\title{
Shot noise and coherent multiple charge transfer in superconducting quantum point-contacts
}

\author{
J.C. Cuevas, A. Martín-Rodero and A. Levy Yeyati \\ Departamento de Física Teórica de la Materia Condensada C-V, Universidad Autónoma de \\ Madrid, E-28049 Madrid, Spain
}

(October 1, 2018)

\begin{abstract}
We analyze the shot noise in a voltage biased superconducting quantum point-contact. Results are presented for the single channel case with arbitrary transmission. In the limit of very low transmission it is found that the effective charge, defined from the noise-current ratio, exhibits a step-like behavior as a function of voltage with well defined plateaus at integer values of the electronic charge. This multiple charge corresponds to the transmitted charge in a Multiple Andreev Reflection (MAR) process. This effect gradually disappears for increasing transmission due to interference between different MAR processes.
\end{abstract}

Typeset using REVTEX 
In the last few years much attention has been paid to the study of shot noise in mesoscopic systems [1,2]. These time-dependent current fluctuations are a consequence of the discreteness of the charge carriers and their measurement can provide information on correlations and charge of the individual carriers not available in usual conductance experiments. In the case of a current $I$ of uncorrelated carriers of charge $q$, the shot noise reaches its maximum value $S=2 q I=S_{\text {Poisson. }}$. This result has been recently used for detecting the fractional $e / 3$ charge carriers by measuring the ratio $S / 2 I$ in the fractional quantum Hall regime [3].

In low-transmitting normal-superconducting N-S structures a doubling of the normal Poisson noise-current ratio has been predicted [4-6] due to Andreev processes where twice the electron charge is transmitted. In S-N-S or S-I-S structures the situation is far more complex. In this case, it is well established that the main processes contributing to the current for subgap bias voltages are multiple Andreev reflections (MAR) [7]. At a given subgap voltage $V$ the current is mainly carried by MAR processes of order $n \sim 2 \Delta / e V$, in which a net charge of ne is transferred. One would then expect an increase of the noise-current ratio roughly as $1 / V$ for decreasing bias. This qualitative behavior has been recently confirmed experimentally by Dieleman et al. [8] for a S-I-S tunnel junction. These authors give an explanation of the observed shot noise enhancement due to MAR within the framework of the semi-classical theory of Ref. [0].

In large tunnel junctions a quantitative comparison between theory and experiment is prevented by unavoidable uncertainties in the junction geometrical structure. On the other hand, there has been in recent years a large progress in the fabrication of superconducting point contacts with a reduced number of conducting channels with rather controllable transmission [9,10]. The subgap structure (SGS) in the I-V characteristics of these systems has been quantitatively described with high accuracy using fully quantum mechanical theories of transport through a single channel [11,12]. One would expect that a similar quantitative agreement between theory and experiment could be reached also in the case of noise.

So far the analysis of current fluctuations in a superconducting single channel contact 
has been restricted to a few special cases. Thus, the excess noise $(e V \gg \Delta)$ for a perfect transparent channel has been obtained in Ref. [13]. The noise has also been analyzed for zero voltage and arbitrary transmission in Ref. [14], while the perfect transmission and finite voltage case has been addressed in Ref. [15].

The aim of this work is to analyze the shot noise for the whole range of transmissions and voltages within the same microscopic model used for the calculation of the current in Ref. [12]. This analysis would allow to answer the question on whether a well defined $q=S / 2 I$ transmitted effective charge can be associated to the processes giving rise to the SGS in the current. We shall explicitly show that this is only possible in the low transmission regime where the effective charge tends to integer multiples of the electron charge $n=1+\operatorname{Int}[2 \Delta / e V]$.

We shall consider the case of a superconducting quantum point-contact (SQPC), i.e. a short $\left(L \ll \xi_{0}\right)$ mesoscopic constriction between two superconducting electrodes with a constant applied bias voltage $V$. For the range $e V \sim \Delta$ one can neglect the energy dependence of the transmission coefficients and all transport properties can be expressed as a superposition of independent channel contributions [16]. Thus, we will concentrate in analyzing a single channel model which can be described by the following Hamiltonian [12

$$
\hat{H}(\tau)=\hat{H}_{L}+\hat{H}_{R}+\sum_{\sigma}\left(t e^{i \phi(\tau) / 2} c_{L \sigma}^{\dagger} c_{R \sigma}+t^{*} e^{-i \phi(\tau) / 2} c_{R \sigma}^{\dagger} c_{L \sigma}\right)
$$

where $\hat{H}_{L, R}$ are the BCS Hamiltonians for the left and right uncoupled electrodes, $\phi(\tau)=$ $\phi_{0}+2 e V \tau / \hbar$ is the time-dependent superconducting phase difference, which enters as a phase factor in the hopping terms describing electron transfer between both electrodes. The normal transmission coefficient, $\alpha$, can be varied between 0 and 1 as a function of the hopping parameter $t$ (see ref. [12] for details). Within this model the current operator is given by

$$
\hat{I}(\tau)=\frac{i e}{\hbar} \sum_{\sigma}\left(t e^{i \phi(\tau) / 2} c_{L \sigma}^{\dagger}(\tau) c_{R \sigma}(\tau)-t^{*} e^{-i \phi(\tau) / 2} c_{R \sigma}^{\dagger}(\tau) c_{L \sigma}(\tau)\right)
$$

The current-noise spectral density is defined as

$$
S(\omega, \tau)=\hbar \int d \tau^{\prime} e^{i w \tau^{\prime}}<\delta \hat{I}\left(\tau+\tau^{\prime}\right) \delta \hat{I}(\tau)+\delta \hat{I}(\tau) \delta \hat{I}\left(\tau+\tau^{\prime}\right)>\equiv \hbar \int d \tau^{\prime} e^{i w \tau^{\prime}} K\left(\tau, \tau^{\prime}\right)
$$


where $\delta \hat{I}(\tau)=\hat{I}(\tau)-<\hat{I}(\tau)>$ is the time-dependent fluctuations in the current.

In the case of a voltage biased superconducting contact both $<\hat{I}(\tau)>$ and $S(\omega, \tau)$ contains all the harmonics of the Josephson frequency $\omega_{0}=2 e V / \hbar$, i.e. $<\hat{I}(\tau)>=$ $\sum_{n} I_{n} \exp \left[i n \omega_{0} \tau\right]$. These quantities can be expressed in terms of non-equilibrium Green functions in a superconducting broken symmetry or Nambu representation $\hat{G}_{i j}^{+-}\left(t, t^{\prime}\right)$ and $\hat{G}_{i j}^{-+}\left(t, t^{\prime}\right)$ where $i, j \equiv L, R$ defined as

$$
\hat{G}_{i, j}^{+-}\left(\tau, \tau^{\prime}\right)=i\left(\begin{array}{l}
<c_{j \uparrow}^{\dagger}\left(\tau^{\prime}\right) c_{i \uparrow}(\tau)><c_{j \downarrow}\left(\tau^{\prime}\right) c_{i \uparrow}(\tau)> \\
<c_{j \uparrow}^{\dagger}\left(\tau^{\prime}\right) c_{i \downarrow}^{\dagger}(\tau)><c_{j \downarrow}\left(\tau^{\prime}\right) c_{i \downarrow}^{\dagger}(\tau)>
\end{array}\right),
$$

and obey the relation $\hat{G}_{i, j}^{-+}\left(\tau, \tau^{\prime}\right)=\left[\hat{G}_{j, i}^{+-}\left(\tau, \tau^{\prime}\right)\right]^{\dagger}$.

Then, the mean current and the kernel $K\left(\tau, \tau^{\prime}\right)$ in the noise spectral density are given by

$$
\begin{aligned}
<\hat{I}(\tau)>= & \frac{e}{\hbar} \operatorname{Tr}\left[\hat{\sigma}_{z}\left(\hat{t}(\tau) \hat{G}_{R L}^{+-}(\tau, \tau)-\hat{t}^{\dagger}(\tau) \hat{G}_{L R}^{+-}(\tau, \tau)\right)\right] \\
K\left(\tau, \tau^{\prime}\right)= & \frac{e^{2}}{\hbar^{2}}\left\{\operatorname { T r } \left[\hat{t}^{\dagger}(\tau) \hat{G}_{L L}^{+,-}\left(\tau, \tau^{\prime}\right) \hat{t}\left(\tau^{\prime}\right) \hat{G}_{R R}^{-,++}\left(\tau^{\prime}, \tau\right)+\hat{t}(\tau) \hat{G}_{R R}^{+,-}\left(\tau, \tau^{\prime}\right) \hat{t}^{\dagger}\left(\tau^{\prime}\right) \hat{G}_{L L}^{-,+}\left(\tau^{\prime}, \tau\right)-\right.\right. \\
& \left.\left.\hat{t}^{\dagger}(\tau) \hat{G}_{L R}^{+,-}\left(\tau, \tau^{\prime}\right) \hat{t}^{\dagger}\left(\tau^{\prime}\right) \hat{G}_{L R}^{-,+}\left(\tau^{\prime}, \tau\right)-\hat{t}(\tau) \hat{G}_{R L}^{+,-}\left(\tau, \tau^{\prime}\right) \hat{t}\left(\tau^{\prime}\right) \hat{G}_{R L}^{-,++}\left(\tau^{\prime}, \tau\right)\right]+\left(\tau \rightarrow \tau^{\prime}\right)\right\},
\end{aligned}
$$

where $\hat{\sigma}_{z}$ is the Pauli matrix, $\operatorname{Tr}$ denotes the trace in the Nambu space and $\hat{t}$ is the hopping in this representation

$$
\hat{t}=\left(\begin{array}{cc}
t e^{i \phi(\tau) / 2} & 0 \\
0 & -t^{*} e^{-i \phi(\tau) / 2}
\end{array}\right) .
$$

In the expression (5) for the kernel we have factorized the two body correlation functions following a mean field BCS decoupling scheme [14. The problem of evaluating the Fourier components of $I$ and $S$ can be reduced to the calculation of the Fourier components of the Keldysh Green functions. An efficient algorithm for this evaluation can be found in Ref. [12. We shall restrict our attention to the zero-frequency dc component of the noise $S \equiv \overline{S(0, \tau)}$ at zero temperature.

Fig. 1 illustrates the behavior of $S$ as a function of $V$ for different values of the normal transmission $\alpha$. For comparison, the dc component of the current is also shown. As can be 
observed, the more noticeable features in the shot noise are: (i) the presence of a strongly pronounced subgap $(V \leq 2 \Delta)$ structure, which persists up to transmissions close to one (in the dc current this structure is only pronounced for low transmissions). (ii) In the low transparency limit the shot noise subgap structure consists of a series of steps at voltages $e V_{n}=2 \Delta / n$ ( $n$ integer) as in the case of the dc current. (iii) For higher transmissions there is a steep increase in the noise at low voltages. (iv) For perfect transmission the shot noise is greatly reduced. (v) In the large voltage limit there is an excess noise with respect to the normal case.

Let us start by analyzing the low transmission regime. In this case it turns out that the electronic transport is well described by a sequential tunneling picture [17] and the current can be written as the sum of tunneling rates for each multiple Andreev process times the transmitted charge, i.e. $I_{0}(V)=e \sum n \Gamma_{n}(V)$ with $\Gamma_{n}=(2 / h) \int d \omega R_{n}(\omega)$. The probability of an $n$ th-order Andreev process $R_{n}$ is given by [12]

$$
R_{n}(\omega)=\frac{\pi^{2} \alpha^{n}}{4^{n-1}}\left[\prod_{i=1}^{n-1}|p(\omega-i e V)|^{2}\right] \rho(\omega-n e V) \rho(\omega) ; \omega \in[\Delta, n e V-\Delta],
$$

where $\rho(w)=|\omega| / \sqrt{\omega^{2}-\Delta^{2}}$ is the dimensionless BCS density of states and $p(\omega) \sim$ $\Delta / \sqrt{\Delta^{2}-\omega^{2}}$ is the Cooper pair creation amplitude. Expression (7) for $R_{n}$ clearly displays the different ingredients in a MAR processes, i.e. it is proportional to the initial and final density of states, to the probabitlity of creating $n-1$ Cooper pairs and to the probability of a quasiparticle crossing $n$ times the interface $\left(\alpha^{n}\right)$. In this regime the shot noise adopts an appealing form in terms of $R_{n}$

$$
S=\frac{4 e^{2}}{h} \int d \omega\left\{\sum_{n=1}^{\infty} n^{2} R_{n}-\left(\sum_{n=1}^{\infty} n R_{n}\right)^{2}\right\} .
$$

This expression corresponds to the fluctuations of a random variable (the current) having a multinomial distribution. This is a generalization of the simple binomial distribution one finds in a N-N contact, which leads to the well known $\alpha(1-\alpha)$ behavior [1.24.4.

This analysis shows that in the limit of vanishing transmission only the lowest order process with a non-zero probability contributes to the current at a given bias voltage. As a 
consequence the effective charge shows a step-like behavior described by the formula $q / e=$ $S / 2 e I=1+\operatorname{Int}[2 \Delta / e V]$. The inset in figure 2(b) shows the effective charge for $\alpha=0.01$ together with the step-like function corresponding to the $\alpha \rightarrow 0$ limit. It is also shown in this figure the comparison between the tunnel approximation given by Eq. (8) and the exact result for a transmission $\alpha=0.1$. Notice that even for this small transmission value there are deviations from the simple step-like behavior which are increasingly pronounced when the voltage is reduced. These deviations are produced by the contribution of more than one MAR process at a given voltage.

As the transmission increases the sequential tunneling picture breaks down due to the interference between different MAR processes contributing to the current at any voltage. The charge quantization found in the tunnel regime progressively disappears and is eventually washed out when approaching perfect transmission. This is illustrated in Fig. 2(a).

For intermediate transmissions the analysis of the shot noise becomes rather involved not only due to the interference between different processes but also to the increasing contribution of fluctuations of higher harmonics of the current. On the other hand, in the perfect transmission limit, the analysis is again simplified due to the absence of backscattering. Within our theory one obtains a simple expression for the shot noise in this limit

$$
S=\frac{8 e^{2}}{h} \int d \omega\left[\sum_{n=0}^{\infty} R_{n}\left(1-R_{n}\right)\right]\left[1+2 \sum_{k=1}^{\infty} \prod_{l=1}^{k}|r(\omega+l e V)|^{2}\right],
$$

where $R_{n}$ are the multiple Andreev reflection probabilities given by $R_{n}(\omega)=\prod_{m=0}^{n} \mid r(\omega-$ $m e V)\left.\right|^{2} ; \omega \in[n e V,(n+1) e V]$ and $r(\omega)=\left(\omega+i \sqrt{\Delta^{2}-\omega^{2}}\right) / \Delta$ is the Andreev reflection amplitude at an N-S interface. This expression can be shown to be equivalent to the result of Averin and Iman in Ref. [15]. The great reduction of noise that can be observed in Fig. 1 for perfect transmission is a consequence of having an Andreev reflection probability equal to one inside the gap.

It is also interesting to analyze the large voltage limit, in which the zero-frequency noise behaves as $\lim _{V \rightarrow \infty} S=\left(4 e^{2} / h\right) \alpha(1-\alpha) V+S_{e x c}$, i.e. the shot-noise of a normal contact with transmission $\alpha$ plus and "excess noise" $S_{\text {exc }}$. The excess noise as a function of transmission 
is shown in Fig. 3. $S_{\text {exc }}$ has the same physical origin as the excess current $\left(I_{\text {exc }}\right)$, which arises from the contribution of the first order Andreev process. One should remark that the approach to the asymptotic value is much slower for the shot noise than for the current. We obtain that at zero temperature $S_{\text {exc }}$ is twice the excess noise of a N-S contact [4] with the same transmission. In particular, this relation yields $S_{\text {exc }}=2 / 5 e I_{\text {exc }}$ for the perfect ballistic case in agreement with Ref. [13].

A word of caution should be said about the validity of this theory in the limit of extremely small bias voltage. In this limit there is another energy scale determined by the inelastic relaxation rate $\eta$ (which is a small fraction of $\Delta$ ) playing a role in the theory (see Ref. [14]). This finite relaxation rate introduces a cut-off in the MAR processes which determines the behavior of current [12] and noise when $e V \ll \eta$. The precise behavior at $V \rightarrow 0$ thus depends on the actual value of $\eta$.

In conclusion, we have analyzed theoretically the shot noise in a single channel SQPC for arbitrary transmission and bias voltage. We have shown that the shot noise can be much larger than the Poisson noise $\left(S_{\text {Poisson }}=2 e I\right)$ due to the occurrence of multiple Andreev reflections in which multiple charge quanta are transferred. In the tunnel regime, the effective charge $q(V)=S / 2 I$ shows a step-like behavior which is a signature of the coherent transmission of multiple electronic charges. Our results are consistent with the available experimental data of Dieleman et al. [8]. These authors used large tunnel junctions for which a mean transmission of $\alpha=0.17$ was estimated. Although a direct comparison with theory is difficult due to uncertainties in the junction structure, the experimental results for shot noise and the effective charge are in qualitative agreement with the results of our Figs. 1-2 for this transmission range. On the other hand, the predictions presented in this letter are amenable to direct experimental test using state of the art techniques for fabricating atomic-size contacts [9,10]. In view of the remarkable agreement between theory and experiments for the current-voltage characteristics found in these systems, one would expect a similar quantitative agreement when measuring current-current fluctuations. 


\section{ACKNOWLEDGMENTS}

The authors would like to thank C. Urbina and E. Scheer for useful discussions. This work has been supported by the Spanish CICYT under contract No. PB97-0044. 


\section{REFERENCES}

[1] For a recent review, see M.J.M. de Jong and C.W.J. Beenakker, in: Mesoscopic Electron Transport, ed. by L.L. Sohn, L.P. Kouwenhoven, and G. Schön, NATO ASI Series E, Vol. 345 (Kluwer Academic Publishing, Dordrecht, 1997).

[2] M. Büttiker, Phys. Rev. B 46, 12485 (1992).

[3] L. Saminadayar et al., Phys. Rev. Lett. 79, 2526 (1997).

[4] V.A. Khlus, Sov. Phys. JETP 66, 1243 (1987).

[5] M.J.M. de Jong and C.W.J. Beenakker, Phys. Rev. B 49, 16070 (1994).

[6] B.A. Muzykantskii and D.E. Khmelnitskii, Phys. Rev. B 50, 3982 (1994).

[7] T.M. Klapwijk, G.E. Blonder and M. Tinkham, Physica B 109\&110, 1657 (1982).

[8] P. Dieleman et al., Phys. Rev. Lett. 79, 3486 (1997).

[9] E. Scheer et al., Phys. Rev. Lett. 78, 3535 (1997).

[10] E. Scheer et al., Nature 394, 154 (1998).

[11] D. Averin and A. Bardas, Phys. Rev. Lett. 75, 1831 (1995).

[12] J.C. Cuevas, A. Martín-Rodero and A. Levy Yeyati, Phys. Rev. B 54, 7366 (1996).

[13] J.P. Hessling et al., Europhys. Lett. 34, 49 (1996).

[14] A. Martín-Rodero, A. Levy Yeyati and F.J. García-Vidal, Phys. Rev. B , 53, R8891 (1996).

[15] D.V. Averin and H.T. Imam, Phys. Rev. Lett. 76, 3814, (1996).

[16] A. Bardas and D. Averin, Phys. Rev. B 56, R8518 (1997).

[17] A. Levy Yeyati et al., Phys. Rev. B 55 R6137 (1997). 


\section{FIGURES}

FIG. 1. (a) Current-voltage and (b) noise-voltage characteristics for different transmissions at zero temperature. The values of the transmission are the same in both panels.

FIG. 2. (a) Effective charge as a function of voltage for different transmissions. (b) Comparison between the exact result (full line) and the tunnel approximation given by Eq. (8) (open circles)

for $\alpha=0.1$. The inset shows the exact result (full line) for $\alpha=0.01$ and the step-like function (dashed line) corresponding to the $\alpha \rightarrow 0$ limit.

FIG. 3. Excess noise as a function of transmission at zero temperature. 


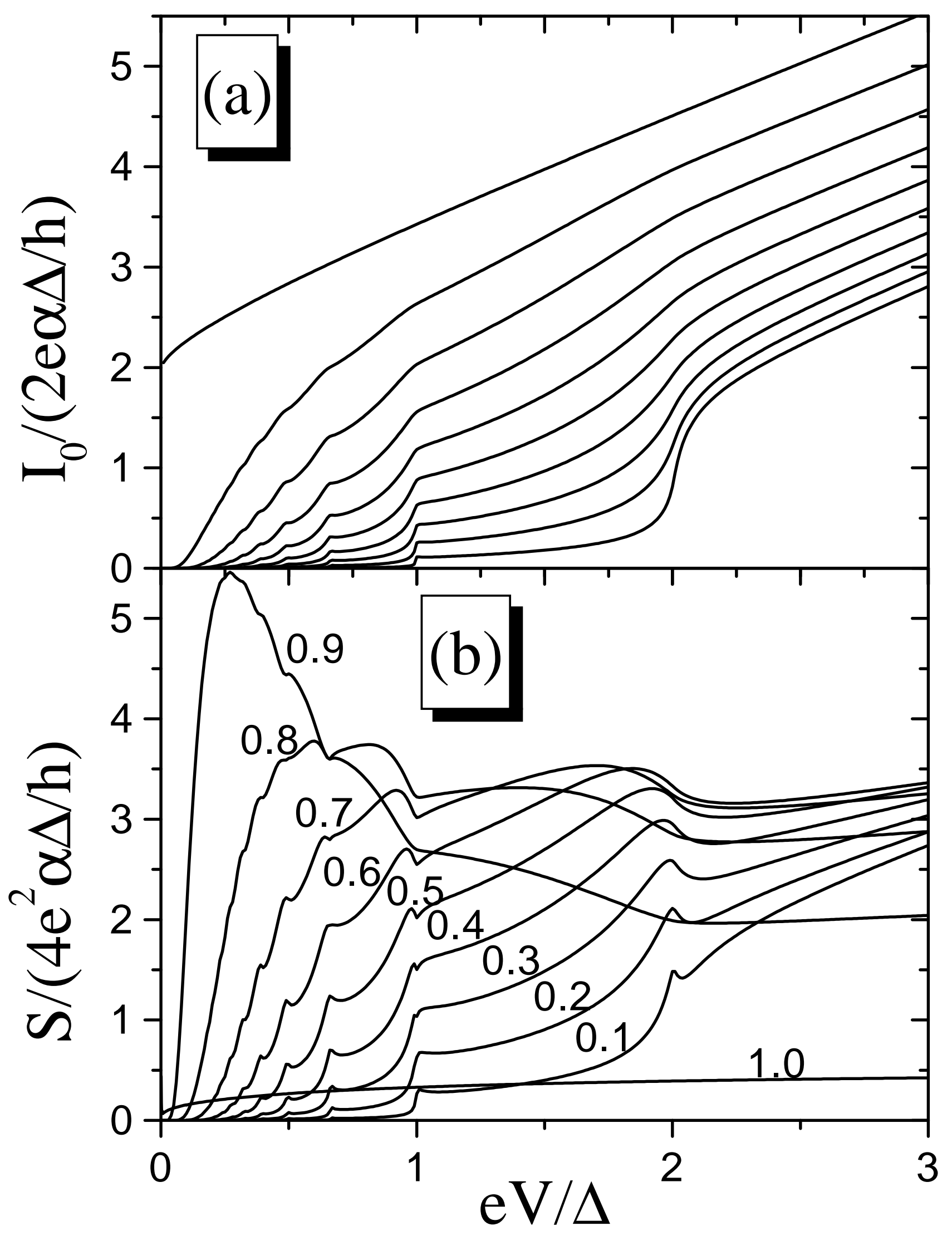



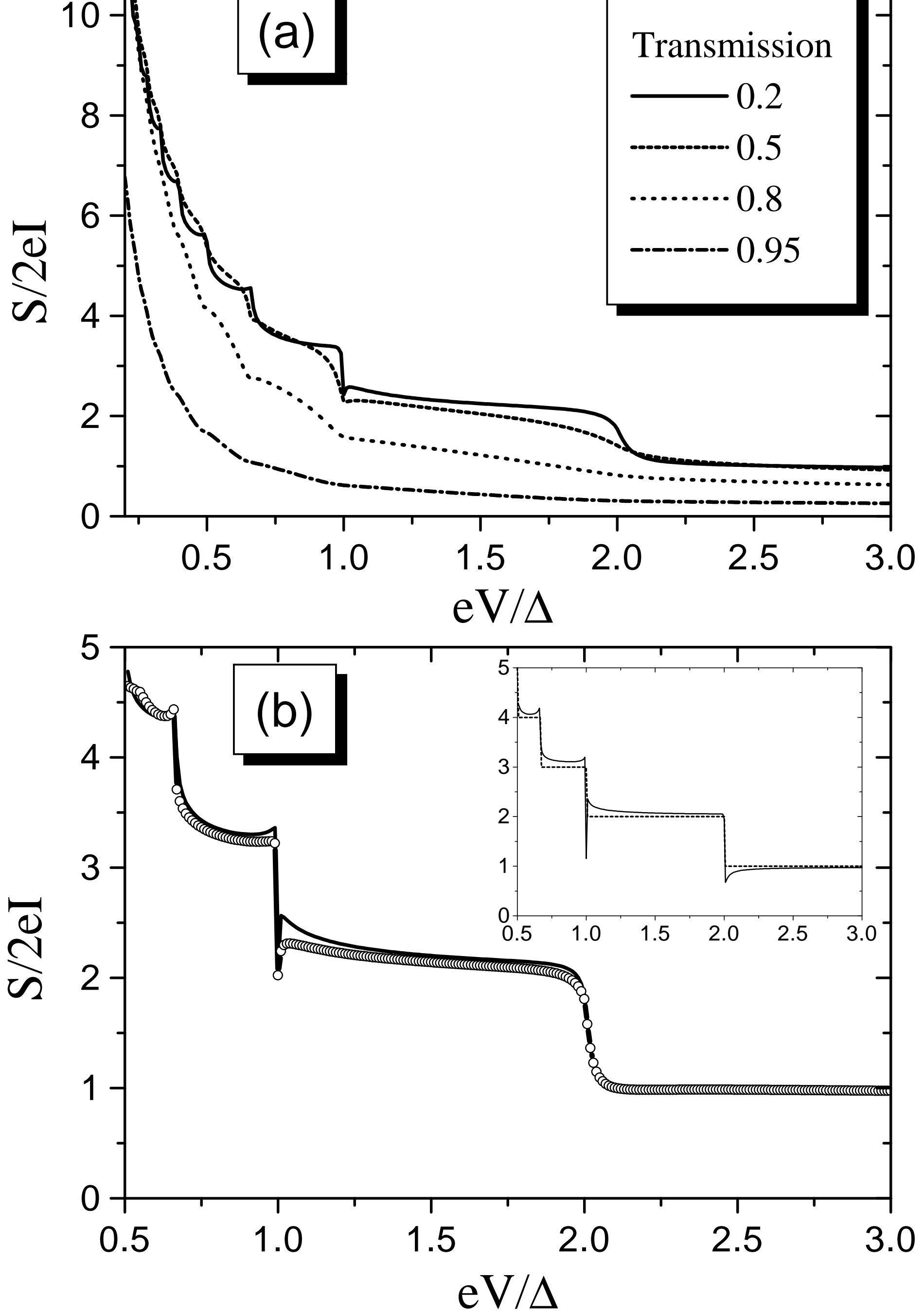


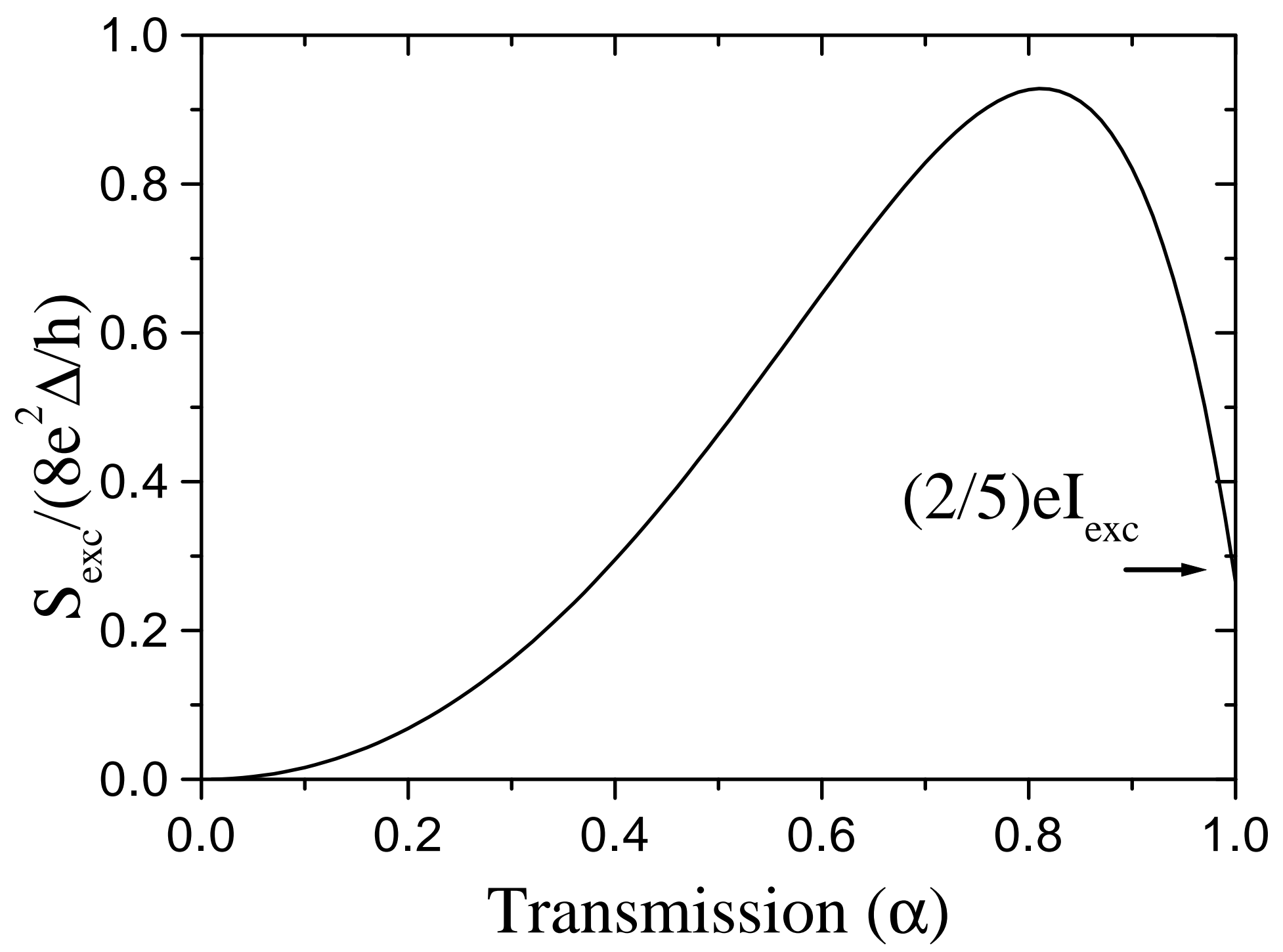

